\title{
A Study on Recent Trends in Artificial Intelligence
}

\section{OPEN ACCESS}

Volume: 6

Issue: 4

Month: April

Year: 2019

ISSN: 2321-4643

Received: 12/03/2019

Accepted: 30/03/2019

Published: 01/04/2019

Citation:

Harris Kumar, D., and V. Renga Boss. "A Study on Recent Trends in Artificial Intelligence." Shanlax International Journal of Management, vol. 6, no. 4, 2019, pp. 33-35.

DOI:

https://doi.org/10.34293/ management.v6i4.373

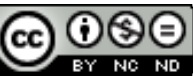

This work is licensed under a Creative Commons Attribution-NonCommercialNoDerivatives 4.0

International License
D.Harris Kumar

Assistant Professor, Department of MBA

KV Institute of Management and Information Studies, Coimbatore, Tamil Nadu, India

\section{V.Renga Boss}

Assistant Professor, Department of MBA

$K V$ Institute of Management and Information Studies, Coimbatore, Tamil Nadu, India

\begin{abstract}
An effective personal assistant should have adaptability, judgment, and technical, team player, customer service \& communication skills, from the olden days personal assistant has been assigned to look after a team. A personal assistant (PA) usually carries out administrative work on behalf of one individual. The role of a PA is to free an executive's time from organizing and administrative tasks so that they can spend maximum time on strategic tasks. Now we are living in the technological emerging world, physical personal assistants are replaced by the virtual assistant, they can be in the form of small device or robots and even it can be a contract that is signed over the Internet that assists in the day to day tasks performed by the human. Artificial intelligence plays an important role in a virtual assistant. One common characterization of intelligent computation is based on the ability to solve a problem. Types of problems requiring intelligence include inference based on acquiring knowledge, reasoning, and acting with uncertain or incomplete information, various forms of perception and learning, and applications to problems such as control, prediction, classification, and optimization. The second characterization of AI computation is based on the underlying mechanism for biological processes it is used to arrive a solution. The primary variant of this category is neural networks and genetic algorithms. This view of AI is necessary even if such techniques are used to compute things that don't appear intelligent in the way of computing. In this article the current trend of Artificial intelligence has been discussed.
\end{abstract}

Keywords: Artificial intelligence, Personal Assistant, Virtual Assistant, Human Intellectual device, Machine Learning, Human Artificial interface.

\section{Content}

An Artificial intelligent system is a system that has similar to a living organism, a coherent set of components and subsystems are placed and programmed to work together to engage in goal-driven activities. In general, an intelligent system can sense and respond to the changing environment that is happening around it gather and store information in its memory; learn from earlier experiences; adapt its behaviors to meet new challenges/tasks and achieve its pre-determined or evolving objectives. The system will start with a set of predefined stimulus-response rules. Those rules will be revised and improved through learning. Anytime when the system encounters a trouble situation, it evaluates and selects the most appropriate rules that are programmed from its memory to act upon the situation. We are privileged generation to live in this era full of technological advancements happening in and around of us, days has been and gone when almost everything was done manually, and now we are in extinction where all the works have been done by machines, software's in PC and other programmed automated process. 
With this advancement artificial intelligence has a special place in all the advancements that are made/ created today. Artificial intelligence or AI is nothing is the science of computers and machines will be developing intelligence like humans being. In this AI all the difficult and complex tasks of humans will be done easily by systems.AI systems help humans to perform the assignments in day to day basis.

\section{Great help for Humans}

The AI systems are more efficient to reduce human efforts in various areas. To perform various sectors in the industry, many are using artificial intelligence to create machine slaves that perform various activities regularly. The artificial intelligence applications le firms to finish the works faster with more accurate results. Error-free and efficient works are the main reason behind artificial intelligence implementation.

According to Deloitte's Global Mobile Consumer Survey, 65 percent of smartphone owners across 16 developed markets have used an application featuring machine learning from 2107 till present. Many of these applications think predictive text and gives route suggestions for users, they are designed to make our mobile experience feel more personalized and convenient to use they are integrated with algorithms, hardware, and the underlying data sets improve over time gradually.

The following chart, based on Deloitte's findings, shows how many mobile users are aware of already using different AI-powered applications on their devices. Predictive text is the most used application involving machine learning, although many of us have probably questioned the (artificial) intelligence of such features based on personal experience with text messages that have gone crooked now.

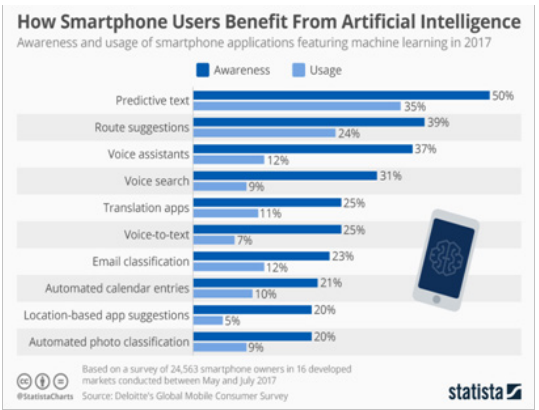

The digital assistant is provided for software service, possibly coupled with a specialized hardware device, such as smart speaker or simply a feature offered on a general purpose computing device. Personal computer, tablet, smartphone or wearable computer (such as a digital wristwatch), which offers some interesting set of the abilities. Most notably answering questions and performing tasks using voice and natural language processing (NLP) backed by artificial intelligence (AI).Some of the popular personal assistants are listed below.

From the above we can understand that Google assistant tops the table followed by Siri,Nina , and Google Now. Google assistant will have access to various aspects like weather, Flight status or places, it searches for you and it has nearly around 200 languages compatible. Siri created by Apple Inc it manage activities through remainders for smooth operations. Cortana created by Microsoft and it is an intelligent personal assistant with location-based remainders at specific date and time. Amazon Echo is hands-free speakers developed by Amazon and it will be controlled by Alexa voice service we can have a voice conversation with Echo and it answers the question by accessing the Internet it can alexa will pick act song as preferred. Nina acts as Automated intelligent assistant used for customer self-services. The future of $\mathrm{Ai}$ is been displayed in the image below, it clearly shows that AI will nearly around 45 million US Dollars will be the approximate revenue that will be generated in various fields.

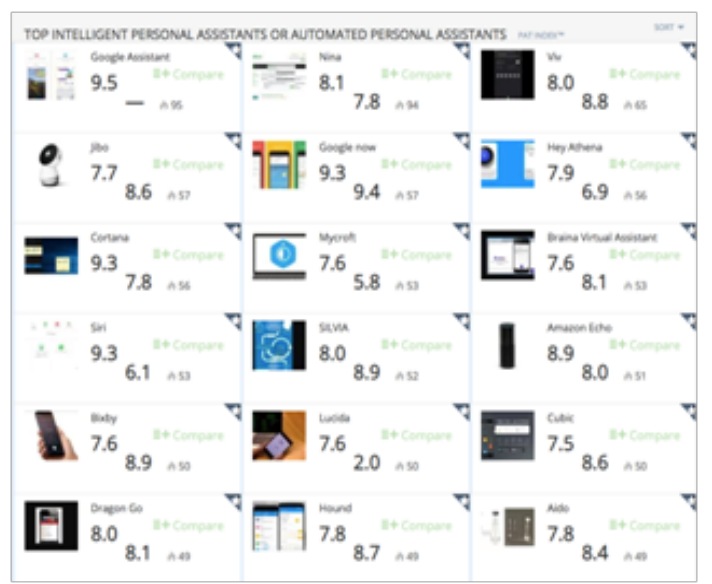




\section{Conclusion}

AI technology becomes part of everyone's life. From little to complex work everyone now using AI to generate leads and remove the work pressure easily. These technologies are so advanced that you don't have to write code for every activity, they will understand the motion of work automatically. As humans, we should be able to balance the technology with manual works or else we will be too much depend upon technology than independent thinking will be reduced in the greater extent. Later from now, we will be the slave for machines and they will be ruling us.

\section{References}

\section{Text Books}

Adelson-Velsky, G.M., Arlazarov, V.L., Bitman, A.R., Zhivotovsky, A.A., and Uskov, A.V. (1970). Programming a computer to play chess. Russian Mathematical Surveys, 25:221-262. Life 3.0: Being Human in the Age of Artificial Intelligence.
Agmon, S., (1954). The relaxation method for linear inequalities. Canadian Journal of Mathematics, 6(3):382-392.

Anderson, J. (1980). Cognitive Psychology and its Implications. W. H. Freeman, New York.

Shai Shalev-Shwartz and Shai Ben-David Understanding Machine Learning: From Theory to Algorithms.

Goodfellow, Yoshua Bengio and Aaron Courville Deep Learning.

\section{Websites}

https://searchcrm.techtarget.com/definition/virtualassistant

https://www.predictiveanalyticstoday.com/topintelligent-personal-assistants-automatedpersonal-assistants/

https://www.computerworld.com/article/3264433/ ai-and-speech-advances-bring-virtual-assistantsto-work.html

https://www.infoworld.com/article/3275569/virtualassistants-dont-need-developers-or-ai.html https://yourstory.com/2018/05/consumer-bankingai-virtual-assistants

\section{Author Details \\ Prof.D.Harris Kumar, Assistant Professor, Department of MBA, KV Institute of Management Information Studies, Coimbatore, Tamil Nadu, India, Email ID: indianharris@gmail.com.}

Prof.V.Renga Boss, Assistant Professor, Department of MBA, KV Institute of Management Information Studies, Coimbatore, Tamil Nadu, India. 